\title{
Anabases
}

ANABASES Traditions et réceptions de l'Antiquité

$4 \mid 2006$

Varia

\section{Les Faits des Romains. Une fortune diverse}

\section{Catherine Croizy-Naquet}

\section{OpenEdition}

Journals

Édition électronique

URL : http://journals.openedition.org/anabases/2907

DOI : 10.4000/anabases. 2907

ISSN : 2256-9421

\section{Éditeur}

E.R.A.S.M.E.

\section{Édition imprimée}

Date de publication : 1 octobre 2006

Pagination : 141-154

ISSN : 1774-4296

\section{Référence électronique}

Catherine Croizy-Naquet, «Les Faits des Romains. Une fortune diverse », Anabases [En ligne], 4| 2006 mis en ligne le 01 janvier 2012, consulté le 20 octobre 2019. URL : http://journals.openedition.org/ anabases/2907 ; DOI : 10.4000/anabases.2907 
Anabases 4 (2006), p. 141-154

\title{
Les Faits des Romains Une fortune diverse
}

CaTHERINe Croizy-NaQUeT

\begin{abstract}
Alors QU'AU XII SIÈCLE, la matière troyenne domine largement la scène historiographique de l'Antiquité, elle se voit en partie concurrencée au tout début du XIII siècle par la matière romaine qui, pour la première fois, s'implante de manière durable dans le paysage historique par le biais de l'Histoire ancienne jusqu’à César et plus encore des Faits des Romains ${ }^{1}$. Composée dans les années 1213-1214 en Île-de-France, cette œuvre anonyme est la première « biographie » consacrée à César en langue vernaculaire, à partir de la compilation de sources latines translatées dans une prose nivelant leurs divergences génériques, formelles, stylistiques et idéologiques. Inachevée ${ }^{2}$, elle s'impose comme le monument écrit de la période césarienne et occupe une place importante dans la production vernaculaire dès le XIII siècle ${ }^{3}$ : en témoignent à la fois la soixantaine de manuscrits répertoriés jusqu’à présent, grâce auxquels elle est transmise
\end{abstract}

1 J. LEEKER, Die Darstellung Cäsars in den romanischen Literaturen des Mittelalters, Frankfurt am Main, éd. Vittorio Klostermann, Analecta Romanica, 1986. La partie romaine de l'Histoire ancienne jusqu’à César n'est pas éditée : voir le manuscrit B.N. fr. 20125 pour les sections Rome I (VII) et Rome II (X-XI) : [ $\left.\mathrm{f}^{\circ} 177 \mathrm{v}^{\circ}\right]\left[\mathrm{f}^{\circ}-183 \mathrm{r}^{\circ}\right]-\left[\mathrm{f}^{\circ} 258 \mathrm{v}^{\circ}\right]-\left[\mathrm{f}^{\circ} 375\right.$ $\mathrm{v}^{\circ}$. Li fet des Romains, compilé ensemble de Saluste et de Suetoine et de Lucan, texte du XIII siècle, éd. L.-F. Flutre et K. Sneyders de Vogel, Droz-Paris, Wolters-Gröningue, 2 t., 1938 ; Slatkine reprints, Genève, 1977.

2 Elle devait relater la vie des douze César, comme l'auteur le précise dans le prologue : «Et comencerons nostre conte principalment a Juille Cesar, et le terminerons a Domicien, qui fu li douziemes empereres "(p. 2, 1. 24-26). Mais, pour des raisons inconnues, ce programme n'a pas été mené à bien.

3 L.-F. FLUTRE, "Li Fait des Romains" dans les littératures française et italienne du XIII au XVI" siècle, Paris, Hachette, 1932. 
dans les milieux scolaires et aristocratiques ${ }^{4}$, et le nombre d'emprunts et de réécritures ou simplement des traces plus diffuses. Il n'est guère surprenant qu'elle ait nourri les textes historiques et, même dans bien des cas, supplanté les autorités latines ${ }^{5}$. Il est moins attendu en revanche qu'elle fournisse leur matière à des textes de nature encyclopédique, épique et romanesque, bien que les frontières génériques ne soient pas fermement établies. Pourtant, l'examen des procédés d'adaptation dans trois d'entre eux, en renvoyant, par un effet spéculaire, une image hybride et complexe des Faits des Romains, explique et justifie une réception riche en contrastes et en signifiances.

Les Faits des Romains réunissent toutes les conditions du succès. Par leur contenu à peu près inédit, ils viennent combler un vide en langue vernaculaire, la vie de César in extenso n'ayant fait jusqu'alors l'objet d'aucun texte spécifique ${ }^{6}$. L'Histoire ancienne jusqu’à César, qui précède de peu, s'interrompt sur les premières opérations de César en Gaule, sans s'être jamais attardée auparavant sur son histoire individuelle, Rome comme entité collective mobilisant exclusivement l'intérêt de l'auteur. En changeant de focale, en centrant son récit sur la figure du chef romain, le compilateur fait de César le personnage principal de l'histoire romaine, dont l'existence, les valeurs et les combats sont intrinsèquement liés à l'évolution de Rome. Dotant le narrateur et le lecteur d'un don d'ubiquité, il unit biographie et histoire dans un mouvement dialectique afin de dresser le panorama complet des faits relevant de la période césarienne. Il varie les points de vue, agence la gamme des temps et met en scène tous les acteurs de l'histoire dont les chefs gaulois et les Romains Pompée et Caton ${ }^{7}$. La nouveauté radicale est la position centrale concédée à César, généralement présenté comme le modèle du conquérant-type aux côtés d'Alexandre ou perçu, dans les premiers textes historiographiques du XIIe siècle, en particulier chez Geoffroy de Monmouth et, avec quelques

4 L.-F. FluTre, Les manuscrits des Faits des Romains, Paris, Hachette, 1932. La quantité de manuscrits est une attestation importante mais insuffisante de la fortune d'un texte. Le plus grand nombre de ces textes n'est d'ailleurs pas édité (citons par exemple $\mathrm{La}$ Chronique de Baudouin d'Avesnes présentée par M.-R. Jung, La légende de Troie en France au Moyen Âge. Analyse des versions françaises et bibliographie raisonnée des manuscrits, BâleTübingen, Francke, Romanica Helvetica, 114, 1996, p. 431 et ss.). Les Faits des Romains sont concurrencés aux XIV e et XVe siècles par le retour aux autorités latines et le mouvement de traduction.

6 Voir notre article "César et le romanz au XII siècle ", Entre Moyen Âge et Renaissance, Regards croisés sur Jules César, Cahiers de Recherches Médiévales, dir. B. Ribémont et B. Méniel, 2006, p. 39-50.

7 Sur tous ces aspects et la différence avec l'Histoire ancienne jusqu’à César, voir notre ouvrage, Écrire l'Histoire romaine au début du XIII siècle : l'Histoire ancienne jusqu'à César et les Faits des Romains, Paris, Champion, NBMA, 1999. 
nuances, chez Wace, comme l'artisan majeur de la conquête romaine et l'ennemi irréductible des Bretons ${ }^{8}$.

Le compilateur réinvestit cette figure peu exploitée littérairement par la compilation de sources prestigieuses, dont l'assemblage, expressément signifié par des références souvent très précises, lui permet d'organiser sa matière. La trame événementielle est calquée sur les grands moments de la vie du chef romain, relatés dans l'ordre chronologique par Salluste, César, Lucain et Suétone. Un flash-back préalable sur la naissance de Rome et sur les institutions romaines met en lumière la continuité des valeurs depuis les origines jusqu'à l'époque du héros et la légitimité de son accès au pouvoir 9 . La convocation d'autres autorités dont Virgile, Ovide, Isidore de Séville, la sainte Escriture..., confirme la crédibilité scientifique irréfutable de l'œuvre et en fait la somme de toutes les connaissances passées et présentes sur la période circonscrite ${ }^{10}$. Le principe de totalisation s'appuie sur une temporalité qui compose le cours historique, touche après touche, en conjuguant la loi de la chronologie à l'étagement synchronique avec la présence de paliers de simultanéité, et en pratiquant digressions, retours en arrière et annonces. Il est gouverné par les exigences d'un exposé véridique et démonstratif dont la prose est l'instrument. Associée d'emblée au postulat de vérité dans des prologues polémiques, cette forme est pensée comme le medium du vrai, comme la mimesis du réel passé ${ }^{11}$, parce qu'elle est prétendûment la transposition fidèle du latin dont elle s'attribue l'autorité.

Comme la garantie d'authenticité émanant de la prose n'est pas l'apanage des Faits des Romains, leur fortune tient surtout à la démarche singulière d'un compilateur qui s'estime autant chercheur ou enquêteur sur le passé qu'écrivain. Il épouse en effet les conceptions des historiens antiques, pour qui la rhétorique est un élément déterminant

8 Geoffroy de Monmouth, Historia Regum Britanniae, éd. N Wright, I. Berne Burgerbibliothek MS. 568, Cambridge, 1984 ; trad. L. Mathey, Histoire des rois de Bretagne, Paris, Les Belles Lettres, La Roue à Livres, 1993. Wace, Le Roman de Brut, éd. I. Arnold, Paris, SATF, 1938-1940 ; Wace's Roman de Brut, a History of the British, ed and transl. J. Weiss, Exeter, University of Exeter Press, 1999. Sur les sources de l'histoire romaine dans ces œuvres, $c f$. Cl. STERCKX, "Les sources du récit de la conquête romaine chez Goeffrey de Monmouth et Robert Wace ", Le Roman de Brut, entre mythe et histoire, textes réunis par Cl. Letellier et D. Hüe, Orléans, Paradigme, Medievalia, p. 89-100. Voir également notre article, "César et le romanz", p. 40 et ss.

9 Faits des Romains, "De la naissance de César à la guerre des Gaules”, p. 5-78; "La guerre des Gaules ”, p. 79-346 ; “ La guerre civile”, p. 347-711 ; “César, maître du monde et sa mort", p. 711-744.

10 Cf. notre ouvrage, p. 67-125.

11 Sur la mise en cause des contes rimés, cf. notre article, "Nus contes rimés n'est verais", Revue des Sciences Humaines, Poésie en procès, textes réunis par Cl. Millet, 276, 4/2004, p. 29-44. 
de l'écriture de l'histoire ${ }^{12}$, et il envisage celle-ci comme l'art de dégager les messages que renferme un passé lointain. Aussi emploie-t-il les techniques épico-romanesques en vigueur dans la littérature contemporaine, avec le souci de projeter aux yeux de son public une comédie humaine à l'échelle du peuple romain au moment où s'achève la République, dont il entend dévoiler l'altérité. Mais cet art ne se départit pas d'une assise scientifique, par l'entremise d'un discours savant dispensé sous la forme de données historiques reconstruites chronologiquement et logiquement, de fiches encyclopédiques avec des excursus sur les mœurs de certains peuples et sur la géographie de l'Orient, de digressions généalogiques ou d'explications étymologiques ${ }^{13}$. Il comprend aussi bien des réflexions philosophiques, religieuses, morales et surtout politiques dans les portraits contrastés de personnages campés pour incarner différents modes de pouvoir, autour de la notion de franchise. Cette combinaison d'approches aboutit à une œuvre, historique dans son intentionnalité, mais multiple dans son contenu, comme si le régime d'historicité ne s'obtenait que par le support de tous les types de discours et par la voie de la somme. Le travail de compilation, achèvement de l'érudition, dépasse en l'occurrence le seul versant historiographique pour englober les différents domaines du savoir et satisfaire les attentes les plus diverses du public, variables selon les moments et selon les lieux ${ }^{14}$.

Les Faits des Romains connaissent d'entrée le succès au XIII siècle. Selon Bernard Guenée, l'œuvre aurait d'abord essentiellement touché le public universitaire ${ }^{15}$. Peu convaincue, Gabrielle Spiegel se demande à juste titre ce que pouvaient bien apprendre d'un texte de vulgarisation les clercs lettrés dont les compétences en latin paraissent aller de soi. Elle suppose donc que la compilation a été reçue par les cours seigneuriales du Nord de la France avides de s'instruire sur le passé romain, dans un milieu socioculturel qui y voit aussi incarnée la situation qu'il est en train de vivre et subir ${ }^{16}$. Des allusions intratextuelles à la réalité contemporaine autorisent en effet le parallèle entre

12 Voir notamment la mise au point de J. Gaillard et R. Martin, Les genres littéraires à Rome, Paris, Scodel, t. 1, p. 108 et ss.

13 On signalera par exemple la description des mœurs des Gaulois et des Germains (p. 220228) ; celle des serpents de Libye (p. 602-613) ; la réflexion sur le sort des âmes après la mort (p. 574), etc.

14 Sur le régime d'historicité des Faits des Romains, voir notre article "Troie et Rome et leurs mises en récit au XIII siècle", colloque international Romance : Dimensions of Time and Designs of History, organisé par J. Whitman and The Center for Literary Studies, The Hebrew University of Jerusalem, 28-31 mai 2006, actes à paraître.

B. GuenÉE, " La culture historique des nobles : le succès des Faits des Romains (XIVe-XVe siècles) ", La noblesse au Moyen Âge, Essais à la mémoire de Robert Boutruche, éd. Ph. Contamine, Paris, PUF, 1976, p. 261-288. 1993, p. 120 et ss. 
César qui œuvre pour le passage de la République à l'Empire et Philippe Auguste qui s'efforce d'imposer son autorité aux grands féodaux ${ }^{17}$. Il n'est guère aisé toutefois de déterminer la prise de position exacte du compilateur à l'égard du chef romain, tant les indices sont cryptés ${ }^{18}$, mais le va-et-vient entre deux mondes rappelle que l'historien n'est pas détaché de son temps et que l'histoire qu'il bâtit selon une topique et selon un style se situe au carrefour de maintes influences culturelles. Par la suite et de façon plus générale, en raison de la vocation de sagesse qui lui est attachée, l'œuvre est reconnue comme un livre d'instruction et de divertissement et trouve légitimement place dans l'apprentissage des enfants. Au-delà, elle a pu séduire les nobles lettrés, un Charles le Téméraire par exemple, qui se la faisait lire avec moult grant plaisir ${ }^{19}$.

À la lecture continue de l'œuvre s'ajoute une réception plus active. Attractive pour des lettrés de toute obédience, la facture originale du texte, avec sa nature transgénérique, s'est prêtée à des emprunts, des reprises, des adaptations ou des subversions, comme l'illustrent trois textes de genre, d'époque et de lieu différents.

Avec le Livre dou Tresor daté du XIII siècle, dont le but est d'instruire celui qui doit gouverner une cité, Brunet Latin rédige en prose vernaculaire une encyclopédie fondée sur de nombreuses sources et des lectures considérables et systématiques d'auteurs anciens et contemporains ${ }^{20}$. Dans le Livre III portant sur la rhétorique et sur la science politique, l'auteur au moment de définir ce qu'est un prologue, ce qu'il doit contenir, ce qui fait ses vices et ses vertus, reprend aux Faits des Romains, en totalité et littéralement, les discours prononcés par César et Caton sur le châtiment à infliger aux coupables de la conjuration conduite par Catilina ${ }^{21}$. Bien que Brunet Latin cite aussi Salluste ${ }^{22}$, la source directe du chapitre est bien la compilation désignée sous le nom d'istoires ${ }^{23}$, malgré de menus changements dépendant sans doute du manuscrit utilisé.

17 Faits des Romains, Comparaison entre les deux gouvernants, p. 18, 1. 29 et ss. : "Quant je lis de Juilles Cesar que Luces Silla l'apeloit le valet mau ceint, si me membre de monseignor Phelipe le roi de France...».

G. Spiegel, Romancing the Past, p. 172 et ss. ; J. M. A. Beer, A medieval Caesar, Genève, Études de Philologie et d'Histoire, 30, 1976, p. 156 et ss. Les deux critiques proposent une interprétation opposée.

B. GuenéE, Histoire et culture historique dans l'Occident médiéval, Paris, Aubier, 1987, p. 277.

L.-F. FLUTRE, "Li Fait des Romains" dans les littératures française et italienne du XIII au XVIe siècle, p. 1-2. Brunet Latin, Li Livre dou Tresor, éd. critique F. J. Carmody, Los Angeles, Berkeley, 1948 ; Genève, Slatkine Reprints, 1998. Sur le projet d'instruire, voir le plan et le texte des derniers livres, p. XXV-XXVI.

Faits des Romains, la conjuration de Catilina, p. 21-55; pour les discours de César et de Caton, voir p. 37-40, p. 40-43 ; Brunet Latin, op. cit., p. 344-352.

Op. cit., p. 344, 1. 13-14 : "Salustes dit ke Decius Sillanus [...] dist premiers sa sentence..." ".

Op. cit., p. 344, 1. 7. Le terme désigne-t-il une œuvre historique ou un texte qui fait autorité en langue vernaculaire? 
Les deux discours sont assortis d'un commentaire de l'encyclopédiste qui délaisse le contenu historique des propos, au profit de leur ordonnancement et de leur habileté démonstrative. Dans un catalogue explicatif, il analyse la technique rhétorique de César, en s'attachant en priorité aux normes du prologue conçues pour convaincre un public hostile ${ }^{24}$. Il compare ensuite ce prologue à celui de Caton qui doit donner aux auditeurs l'envie de savoir ce k'il voloit dire por retraire les de la sentence Cesar, selonc ce que li mestres devise ci derieres la u il enseigne la diversité des prologues ${ }^{25}$. La confrontation de deux discours opposés dans leurs revendications - César est contre la peine de mort, Caton la réclame - découvre les ressorts de la persuasion et définit les moyens de persuader son auditoire. Dans cet emprunt direct, Brunet Latin prend pour argent comptant les discours recomposés par le compilateur à partir de Salluste, sans interroger leur authenticité historique, étrangère ou extérieure à sa démonstration, et les considère comme la vivante expression de la rhétorique du prologue dans la langue vernaculaire, celle de son public. Un tel parti pris souligne le statut d'autorité acquis par les Faits des Romains, qui dispense un Brunet Latin séduit par la langue française de retourner à l'original latin. Il met également en évidence l'adaptabilité et la malléabilité de la prose française, capable d'intégrer et d'assimiler les canons de la rhétorique latine.

Un siècle plus tard, un autre Italien, Nicolas de Vérone, se révèle sensible à la rhétorique mais aussi à la teneur historique des Faits des Romains ${ }^{26}$. En 1243, il fait le récit de la Pharsale, dont ne subsiste qu'un seul manuscrit, en reprenant les deux chapitres que le compilateur consacre à cet épisode majeur de la guerre civile ${ }^{27}$. Le texte délivre quelques éléments sur l'auteur et sur son commanditaire versé dans les arts et les lettres, Nicolas 1er d'Esté, prince de la cour de Ferrare, dont le rayonnement culturel reste limité dans la première moitié du XIVe siècle 28 :

Op. cit., p. 347-348 : il disserte sur sa tactique pour donner as ö̈eurs talent de savoir et d'oür ce kill voloit dire, en se servant de covertures et de moz dorés et en respectant les différentes étapes : s'attirer la bienveillance, passer au sujet (le fait), au devisement (c'est-à-dire rappeler les positions des uns et des autres), au confermement (ajouter des preuves à son parti) puis au deffermement (réfutation des objections de ses adversaires), avant de conclure sur une sentence.

Op. cit., p. 351-352.

26 L.-F. FLUTRE, "Li Fait des Romains " dans les littératures française et italienne du XIII au XVIe siècle, p. 112-123.

Nicolas de Vérone, La Pharsale, éd. H. Wahle, Ausgaben und Abhandlungen aus dem Gebiete der Romanischen Philologie, Veröffentlicht von E. Stengel, 80, Marburg, 1888. Faits des Romains, p. 494-567 (III e partie, chap. 13, \$ 23, 1. 31). Voir R. SPECHT, Recherches sur Nicolas de Vérone, Contribution à l'étude de la littérature franco-italienne du quatorzième siècle, Berne, Francfort/Main, Peter Lang, Série XIII, Publications Universitaires Européennes, Langue et littérature française, 1982. Le chapitre 4 est consacré à la Pharsale, p. 111-169. 
Et ce qe çe vous cont dou feit des Romanois

Nicholais le rima dou païs veronois

Por amor son seignor, la flor des Estenois,

Corant mil e troi cent ans e qarante trois. (vv. 1934-1937)

Les Faits des Romains qui sont cités (v. 28, v. 1934) constituent la source effective de la relation, même si l'autorité invoquée est la Pharsale de Lucain, dans des expressions de style formulaire comme mes si cum dit Lucan (v. 40) ou selong l'ancien autor (v. 1932). D'après René Specht, l'auteur n'est pas un lecteur de Lucain et les quelque douze allusions au texte latin pourraient provenir d'un manuscrit des Faits des Romains accompagné de gloses 29 .

Source directe de la Pharsale, les Faits des Romains n'en subissent pas moins un profond remaniement. Selon les termes du prologue, Nicolas de Vérone annonce en effet traiter dans le genre épique un sujet qui n’appartient à aucun des cycles traditionnels dont son public est féru. À ses yeux, la matière romaine, avec la bataille de la Pharsale, mérite un traitement analogue, parce qu'elle s'avère aussi héroïque que tous les grands combats qui rendent le passé antique mythique :

Qar la plus fere zotre ce li ferai entandre

E la gregnor bataille e le greignor contandre

Che fus davant e pois qe Deu se laisa pandre.

Ne ferent tiel bataille li Greçois a dexandre

A port [la] devant Troie, quant l'allerent a prandre,

$\mathrm{Ne}$ anc le roi Porus vers le roi Alexandre,

Comant fist en Thesaile - selong qe puis comprandre,

Le buen Julius Cesar... (vv. 3-11)

Avec la mise en rime, l'histoire romaine est introduite dans l'univers culturel épico-romanesque familier aux zantis de cuer qui Des zouses trepasees vont feisant demandance (vv. 20-22), et peut être ainsi diffusée et enseignée sans le soutien matériel de l'écrit/du livre 30 :

L'en li conte de Hector e de sa convenance,

De Porus, d'Alixandre e de lour asemblance,

De Zarlle, de Roland e de cil de Maiance

E des autres autor ond ne faiz recontance,

Pour ce qe rimé sont selong lour proveance.

Mes dou feit des Romeins ne pooit por certance

Nul conter bien a pont la droite sentance $[\ldots]$

Or le vous veul rimer por tele destinance

30 Le seigneur peut même s'en faire réciter des passages pour se divertir et s'instruire, lorsqu'il est à cheval (v. 32-33). 
Qe cil qe por ma rime l'aura en remembrance

Le pora dir sens livre et sens nulle pesance

Et de falir l'istoire ja non aura dotance. (vv. 23-29 ; vv. 34-37)

Le prologue a valeur performative, puisqu'il enclôt déjà les ingrédients de la chanson de geste : la présence d'un auditeur bien identifié, l'alliance avec les jongleurs appelés à chanter son poème et les lieux communs que sont la vocation édifiante de sa chanson et la communauté des qualités que partagent les acteurs de la Pharsale avec des héros comme Alexandre et Charlemagne.

En dépit du mystère qui l'entoure, le choix délibéré et exclusif des Faits des Romains et non point d'autres récits comme l'Ystoire de Jules César ${ }^{31}$, trouve sa justification dans les combats que le compilateur marque au coin de l'épique ${ }^{32}$. Le fonds latin de Lucain est ainsi déjà médiatisé et aménagé en langue vernaculaire et la nature d'un récit oscillant entre l'histoire et l'épique, l'histoire et la fiction, facilite la littérarisation de la matière que Nicolas de Vérone systématise et régule dans sa mise en vers, au risque de la délester de sa valeur historique.

Le glissement de l'histoire vers la geste se fait par l'alexandrin et, plus rarement, par le décasyllabe. Le respect scrupuleux des règles de versification dénote l'intention de consigner les hauts faits dignes de figurer dans la mémoire collective et celle de célébrer les grands hommes qui en sont les acteurs 33 . L'auteur délaisse d'ailleurs les vers ou fragments de vers et les deux strophes inventés par le compilateur Faits des Romains, en raison de leur aspect fantaisiste qui devait effaroucher le conservateur en lui ${ }^{34}$. Son application scolaire se vérifie dans le travail des laisses. Élaborées suivant le découpage en paragraphes et en chapitres dominant les Faits des Romains, les laisses de longueur variable répondent au schéma établi avec un vers d'intonation initial souvent sophistiqué. Nicolas soigne leur enchaînement, en jouant des anticipations, reprises et transitions 35 .

Jean de Thuyn, Li Hystore de Julius Cesar, éd. Fr. Settegast, Halle, Niemaeyer, 1881.

L'exemple le plus suggestif concerne les hauts faits de Domitius traités à la manière épique dans les Faits des Romains (p. 531-532) : voir notre article "Quand la fiction se mêle à l'histoire : un combat dans les Faits des Romains", Cahiers de Recherches Médiévales. Le choix de la prose (XIII'-XVe siècles), 5, 1998, p. 55-68. Le passage est repris dans une même veine par Nicolas de Vérone, mais avec une systématisation du mode épique par la voie du vers (v. 1540-1555).

Voir par exemple D. BouteT, La chanson de geste, Paris, Puf écriture, 1993 ; sur la versification, p. 101 et ss.

34 Voir K. Sneyders-Vogel, "Les vers dans les Faits des Romains", Mélanges Salverda de Grave, 1933, p. 293-305; notre communication "L'histoire romaine, entre vers et prose" au colloque Écrire en vers, écrire en prose, Une poétique de la révélation, Université Paris XNanterre, 23-25 mars 2006, actes à paraître dans Littérales.

35 R. SPECHT, Recherches sur Nicolas de Vérone, p. 124-125. Nicolas peut réutiliser les hémistiches tels quels (v. 1491 et ss.), ou en les modifiant (v. 2083 et ss.) ; il joue sur l'ordre des mots pour répondre aux besoins de la rime (v. 1312 et ss.) ou recourt à des synonymes (v. 2084 et v. 2086). Sur les effets de ces techniques dans la structuration du récit, voir D. BOUTET, La chanson de geste, p. 159 et ss. 
Les procédés rhétoriques et stylistiques sont également très conventionnels. À partir de phrases isolées empruntées aux Faits des Romains, la bataille de Pharsale se déroule en un affrontement collectif, puis en une série de combats singuliers (p. 140), au moyen des motifs narratifs et rhétoriques propres à la chanson de geste. Des vers comme Dou chief jusqe l'arçon l'a fendu por moités (v. 1098) ou Dou cef le porfendi jusqe pres la mamelle/Mort l'abati a terre... (vv. 1226-1227) doivent avoir une résonance familière pour le public. De même en va-t-il pour les éléments stéréotypés qui inscrivent les combattants dans la tradition épique 36, Pompée par exemple: Quant fu vestus, il ne samble -, ce cuit - /Fil a borçois ni de foble conduit,/Mes plus ardis que lion engluit (vv. 364-366). S'ajoutent les formules topiques comme la veïssiez 37 (v. 362) et les figures de rhétorique dont la répétition, la synonymie, l'hyperbole ${ }^{38}$... Mais la stylisation volontaire de l'événement aboutit à lui faire perdre les singularités historiques que le compilateur cherchait à débusquer. Ce dernier exploitait les typologies épiques et romanesques afin d'actualiser le récit précisément informé des batailles et de dégager la véritable personnalité des héros dans des portraits fouillés et nuancés, sans manichéisme, sans volonté d'héroïcisation systématique. Chez Nicolas de Vérone, la généralisation de l'épique, qui s'accompagne d'un processus d'idéalisation et de célébration à travers un répertoire de comparaisons convenues ${ }^{39}$, a pour effet de simplifier, voire de déproblématiser l'histoire romaine.

Le changement générique induit une recomposition de la matière. S’il reste fidèle au canevas des faits, Nicolas élimine ce qui n'a pas directement trait à l'action principale, tout en conservant les développements épiques inventés par le compilateur auxquels est accoutumé l'auditoire ${ }^{40}$. La complaisance pour la théâtralisation explique aussi bien l'empreinte romanesque plus ponctuelle, que l'auteur revendique lorsqu'il évoque les romans antiques ou les batailles de Thebes e de Troye et d'Egipte... (v. 956958). À l'occasion de la fuite de Pompée et de Cornélie, il laisse les époux exprimer

36 Sur les sens à donner à ces termes et leur classification, voir J.-P. MARTIN, Les motifs dans la chanson de geste. Définition et Utilisation (Discours de l'épopée médiévale), Centre d'Études Médiévales et Dialectales, université de Lille III, 1992.

37 La formule retrouve d'ailleurs sa vertu première de visualisation que son emploi en vers comme en prose a quelque peu fait disparaître au XIII siècle.

38 Cf. R. SPECHT, Recherches sur Nicolas de Vérone, p. 133 et ss. Sur ces techniques, consulter aussi D. BOUTET, La chanson de geste, p. 101-158.

39 Voir le relevé de R. SPECHT, Recherches sur Nicolas de Vérone, p. 142-143; on remarquera, entre autres, la mention des batailles de Thèbes et de Troie (v. 957) et celle du personnage d'Alexandre (v. 8, 24, 108-109, 175 et ss., 1384 et ss., 1622 et ss.). Ces comparaisons dont on peine à définir l'origine tant elles sont vagues s'ajoutent à celles qui émaillent les Faits des Romains et concernent les familles républicaines : Faits des Romains, p. 515/Pharsale, v. 775-779 ; Faits des Romains, p 536/Pharsale, v. 1820-1825 ; Faits des Romains, p. 542/Pharsale, v. 2033-2036.

40 R. SPECHT, Recherches sur Nicolas de Vérone, p. 126-133. Est ainsi omis tout ce qui concerne le destin futur du fils de Pompée (Faits des Romains, p. 495, 1. 22-24). 
longuement leurs états d'âme ${ }^{41}$, sans pour autant soustraire leur histoire d'amour à l'action épique d'ensemble ${ }^{42}$. Il use de la veine romanesque pour mettre en scène les préoccupations singulières des individus plutôt que pour rendre compte, comme dans le roman, des changements qui les affectent. La littérature ne vise qu’à attendrir le public par un art qui lui parle et qu'il maîtrise bien, alors que, dans les Faits des Romains, elle est le laboratoire d'une réflexion sur la place de l'amour et du pouvoir dans la société : les couples, et pas seulement celui de Cornélie et Pompée, sont dépeints et pensés à l'aune de la tradition courtoise ou du moins des interrogations qu'elle suscite ${ }^{43}$. La référence à Troie et à Alexandre contribue encore à réfléchir le rôle plus ou moins opérant ou marginal de la littérature dans les deux textes : dans les Faits des Romains, elle est un élément structurant le parcours politico-hérö̈que de César ${ }^{44}$; chez Nicolas de Vérone, elle est une présence topique, surtout ornementale.

L'auteur italien passe en outre sous silence les réflexions abstraites et les commentaires d'ordre moral qui rythment avec plus de sobriété que chez Lucain la compilation en prose ${ }^{45}$. Si le vers épique ne possède pas le caractère conceptualisant, intellectuel et explicatif de la prose, s'il est plus dans la "monstration " que dans la démonstration, il est néanmoins porteur d'un imaginaire propre et d'une conception du monde et du temps de l'histoire ${ }^{46}$. Or, il ne semble pas apporter de vue personnelle sur la bataille de Pharsale ni renouveler les données de la source par un approfondissement des circonstances et des enjeux qu'elle représente pour l'histoire romaine. Moins encore que son prédécesseur, Nicolas de Vérone ne s'interroge sur les causes de la guerre civile et de la défaite de Pompée ; tout juste invoque-t-il des facteurs surhumains, un dieu ou des dieux, et fait-il appel à la figure commode et protéiforme de Fortune ${ }^{47}$. Il modifie peu le portrait des protagonistes en conflit pour sauvegarder la Franchise de Rome, qui est remise en cause par la volonté dictatoriale de César, sinon en banalisant leurs traits héroïques sur le mode épique : Le buen Julius Cesar ne se bat que par suen honuor

41 Faits des Romains, p. 540-573; Pharsale, v. 2079 et ss.

42 Cf. D. BOuTET, La chanson de geste, p. 215 et ss.

43 Cf. notre ouvrage, p. 252-257.

44 "Alexandre et César dans les Faits des Romains", The Medieval French Alexander, ed. D and S. Maddox, New York, State University of New York Press, Albany, 2002, p. 161-174. Cf. R. SPECHT, Recherches sur Nicolas de Vérone, p. 128 et ss. Disparaissent par exemple les réflexions de Pompée sur son destin et sur le rôle de Fortune : Faits des Romains, p. 546, 1. 6-13/Pharsale, v. 2172-2181.

46 Voir J.-P. MARTIN, “La chanson de geste comme construction de la mémoire ”, colloque international Romance : Dimensions of Time and Designs of History, organisé par J. Whitman and The Center for Literary Studies, The Hebrew University of Jerusalem, 28-31 mai 2006, à paraître.

47 Sur la place de Fortune dans les Faits des Romains, cf. J.M.A. BeER, A medieval Caesar, p. 195 et ss., et chez Nicolas de Vérone, voir R. SPECHT, Recherches sur Nicolas de Vérone, p. 145 et ss. 
defandre/Vers Ponpiu le Roman, quand le cuidoit sourprandre (vv. 10-11). Désireux de le faire entrer dans le monde de la chanson de geste, il est très favorable à l'endroit de César, qu'il apparente à son héros le plus emblématique, Charlemagne. Il dénature ce faisant les Faits des Romains, qui prive le chef romain d'une aura épique contraire à son pragmatisme ${ }^{48}$.

À faire une version en vers qui entre dans le genre des chansons de geste, et plus accessoirement des romans, à ranger César dans la galerie des héros épiques, Nicolas de Vérone tourne résolument le dos à la démarche historicisante de son prédécesseur. Ce dernier comptait rendre au plus juste et au plus près le passé césarien, par une écriture novatrice, miroir d'une conscience historique attentive à faire la part entre ce qui fut et ce qui est, une conscience historique soucieuse de capter l'altérité, au lieu de projeter ses propres valeurs dans le passé. L'auteur italien défait ce que le compilateur a patiemment tissé, une histoire vraie qui échappe, par sa forme inédite et par un contenu encore mal connu, aux affabulations dont sont porteurs les textes en vers, si l'on en croit les contempteurs des contes rimés. En dépit de la sagesse intrinsèque qui distingue la matière romaine, sa version de la Pharsale fausse dans son essence même le récit des Faits des Romains: elle trahit une nette déperdition de ses apports historiques et de la richesse rhétorique au nom de normes établies, de même qu'un retour en arrière dans la réflexion et la composition de l'histoire. L'apport essentiel de Nicolas de Vérone est de déférer aux exigences de la performance orale, dans la perspective du divertissement et de l'enseignement, sans guère apporter de nouveauté ni entraîner de progrès « historique » notable dans la perception de la matière romaine.

La démarche d'un Brunet Latin, reprenant terme à terme un court passage très codé rhétoriquement, et celle d'un Nicolas de Vérone, mettant en vers un épisode célèbre, donnent une juste idée de la reconnaissance dont bénéficient les Faits des Romains comme anthologie par le poids de leur littérarité ou comme autorité historique au point d'éclipser les sources latines. Mais la réception prend une autre allure quand l'œuvre devient une manne d'informations avérées sur César, dans laquelle puiser à volonté sans la moindre attention à ses propriétés littéraires et historiques. Dans le roman Renart le Contrefait, composé dans la première moitié du XIVe siècle, la compilation en prose sert de manuel d'histoire à un clerc de Troyes qui a dû renoncer à la cléricature pour cause de concubinage et qui, après avoir fait du commerce, a mis à profit sa retraite pour rédiger un roman ${ }^{49}$. L'objet d'un récit qui l'aide à combattre l'oisiveté et se distraire n'est pas d'imiter Renart, mais de se contrefaire à lui pour dire par escrit convert/ce qu'il n'osoit dire en appert (vv. 121-122), dans le dessein de moraliser et

48 Voir notre ouvrage, p. 243 et ss.

49 L.-F. FlUTRE, "Li Fait des Romains " dans les littératures française et italienne du XIIIe au XVIe siècle, p. 101-111. Renart le Contrefait, éd. G. Raynaud et H. Lemaître, Paris, SATF, 1914, 2 vol. 
d'édifier le public. Passant en revue les états du monde, l'auteur s'y livre à une satire de la noblesse et du clergé et, en contrepoint, à un éloge du milieu bourgeois et du travail à travers les personnages de Raison et de son alliée Nature, antagoniques à Renart. Les Faits des Romains sont inclus dans la deuxième branche de la seconde édition du roman datée de 1342, où sont ajoutés maints passages théologiques, hagiographiques, historiques et scientifiques, ainsi que des considérations morales exprimées par des lieux communs. La deuxième branche commence par des péripéties au terme desquelles Renart est condamné sans jugement par Lion. Après une intervention de Grimbert en sa faveur, le goupil prend la parole, faisant montre d'une érudition confuse qui n'empêche pas Lion de l'encourager à poursuivre :

Regnard, foy que doy verité,

De Julius Cezar me compte $[\ldots]$

Or me compte la verité

De toute sa nativité,

Desquels il fu et de quel pris.

Par renoumee moult le pris ;

Selonc que l'ay ouy prisier,

On le doit moult auttorisier. (vv. 20454-20462).

Dans l'abrégé d'Histoire universelle qui s'ensuit, la période césarienne se situe dans le fragment réservé aux quatre royaumes antiques, lui-même subdivisé en séquences : la fondation de Rome, les rois, la vie de César, la conjuration de Catilina, la suite de la vie de César et le commencement du règne d'Auguste. Dans ce panorama de l'histoire depuis les origines jusqu'au couronnement de Philippe VI de Valois en 1328, seule la vie de César est issue des Faits des Romains qualifiés d'istore, terme générique qui paraît en désigner sans grande originalité le contenu historique et accréditer sa portée savante ${ }^{50}$. Encore le romancier procède-t-il à une sélection au sein même de la compilation : il néglige le récit de la guerre des Gaules et de la guerre civile, expédié en quelques vers, alors qu'il est fondamental pour apprécier la trajectoire du chef romain ${ }^{51}$, et il préfere Salluste, plus fiable que le compilateur ?, dont le nom est signalé à plusieurs reprises $(20961,21047,21260)$, pour la conjuration de Catilina.

La disparité et l'usage apparemment arbitraire des sources traduisent la grande liberté de l'auteur à l'égard de son matériau de travail, rendue plus manifeste encore, dans le cas des Faits des Romains, par le passage de la prose au vers octosyllabique à rimes plates. Le changement de forme masque l'influence effective et réelle de la compilation qui se lit, pour l'essentiel, dans la similarité des interprétations et dans la reprise

501726 vers proviendraient des Faits des Romains. L'auteur leur emprunte les premiers chapitres (p. 8-20) ; ce qui concerne les mœurs de César sous la forme d'un abrégé et le récit de sa mort (p. 711-744). Voir note 8 sur le plan de l'ouvrage. 
d'erreurs de traduction imputables au prosateur ${ }^{52}$. Pour répondre aux attentes de Lion, le clerc champenois/Renart recentre le parcours biographique sur la naissance et le lignage du héros, et sur la restitution minutieuse des étapes de sa mort 53 . Réduite au portrait partiel et partial du grand homme, l'histoire se voit dévaluée dans sa mission de restituer le réel passé, au profit de finalités démonstratives mais aussi édifiantes. Le narrateur, en moralisateur plutôt qu'historien, profite en effet de l'histoire du héros pour analyser la mentalité de son époque et condamner le peu d'estime dans lequel sont tenus les gens de mérite par ses contemporains (v. 20756 et ss.). La figure de César est prétexte à dénoncer la société de son temps, fustiger les mœurs dépravées et la conduite des nobles et des clercs. Elle sert aussi à louer la France au détriment de Rome dont il est le représentant le plus célèbre, la France qui est ore en haut montee, parce que N'est Dieu tant amé come en France, Si bien servis ne sy doubtés (vv. 21627). Loin d'être contradictoires, ces deux interpolations, entre nostalgie d'un âge d'or et confiance en l'avenir, prouvent que la rédemption et le Salut de l'homme et/ou d'un peuple ne peuvent se gagner que par la foi en Dieu. Ainsi, tout grand homme qu'il soit, César, Qui tant fu orguilleux et fiers/Il fist a maint copz vitupere. (vv. 20447-20451), est voué à déchoir du fait de ses défauts, surtout de son orgueil et de sa démesure, et ne peut s'amender faute des lumières de la foi.

Dans Renart le Contrefait, l'histoire est dessaisie de sa vocation de connaissance du passé. La grille morale qui dicte des choix fragmentaires dans la somme d'une vie en infléchit la perception et en modifie en profondeur la tonalité par la récriture. Se coulant dans le moule de l'octosyllabe, medium favori des romans, l'auteur en reprend aussi les techniques et les figures, descriptions, discours, répétitions, qu'il applique à la trame des Faits des Romains ${ }^{54}$. La vie du chef romain est conçue dans l'esprit du roman, moins pour divertir que pour s'imprimer dans la mémoire de l'auditeur à titre d'exemple des limites irréductibles de l'homme. Il est paradoxal de laisser à Renart le soin de reformuler la vie de César à qui il ressemble par son intelligence et par son art de la manipulation. Le goupil, «maître hypocrite et concurrent de Fortune » selon les termes d'Armand Strubel ${ }^{55}$, principe perturbateur de la création et antithèse du divin,

52 Par exemple, le v. 20511 reprend le contresens des Faits des Romains sur les mots latins Flamen Dialis (p. 8, 1. 12-13) ; le v. 20534 celui sur l'adjectif latin praetextatus, pris pour un nom propre (p. 8, 1. 17) etc. Cf. L.-F. FluTRE, " Li Fait des Romains " dans les littératures française et italienne du XIII au XVIe siècle, p. 102 et ss.

53 Il conviendrait de prendre la mesure de cette récriture, en la confrontant à la représentation d'autres héros comme Alexandre par exemple : l'épisode de la rencontre de l'empereur avec le peuple indien de bons sauvages, guides spirituels qui l'incitent à la sagesse, est traité sur un mode satirique : cf. C. GAULLIER-BOUGASSAS, "Alexandre et les Brahmanes dans les Romans d'Alexandre français, du XII au XVe siècle ", Le Moyen Âge, 106, 2000, p. 467-493.

54 Le romancier dramatise ainsi le conflit entre le Romain Pison et César (v. 20857-20880) ébauché en quelques lignes dans les Faits des Romains (p. 11-12).

A. Strubel, La Rose, Renart et le Graal, Paris, Champion, NBMA, 1989, p. 229. 
parait, en effet, détenir le savoir historique et perdre le temps du récit sa capacité de nuisance. Sa présentation du chef romain reste surtout informative et, somme toute, en-deça de la veine satirique. Renart, en l'espèce narrateur, est le masque que prend l'écrivain désireux de parler sans se dévoiler et de retracer les événements de l'histoire, au rythme du progrès et de la décadence, au travers d'exempla, dont celui réservé à César. Proche à certains égards du Roman de la Rose de Jean de Meung ${ }^{56}$, le catalogue historique laisse deviner en abyme les succès et les échecs d'un Renart qu'il est impossible de vaincre définitivement, comme il est impossible à la société de se stabiliser dans le bien ; il s'accompagne d'une déploration sur la folie des hommes et d'une dénonciation de l'art de la feinte qui gouverne tout, et implique un éventail de règles de conduite d'ordre moral et religieux afin de préparer et d'accomplir son Salut.

Dans un tel contexte, la compilation est dépossédée de son identité par une encyclopédie moraliste qui fait de l'histoire, comme de la théologie et de la science, le moyen de peindre la diversité du monde. Elle est l'instrument d'une démonstration, un livre d'exemple plutôt qu'un récit du passé et voit sacrifiée l'ambition qui était la sienne de faire un compte-rendu exhaustif de la période césarienne et de s'ouvrir à l'altérité romaine.

Le parcours de ces textes d'époques et de genres différents exprime à rebours la complexité des Faits des Romains qui, par leur caractère composite, engendrent une réception à multiples entrées. Dans les trois textes, qui ne sont qu'un infime témoignage de l'influence que l'œuvre a exercée par ailleurs, les modalités d'adaptation montrent un affaiblissement de sa portée initiale et une ignorance du progrès décisif qu'elle a permis de réaliser dans la fabrique de l'histoire. Il s'agit là, il est vrai, de textes plus « littéraires » qu'historiques, de textes pour lesquels l'histoire est le maillon d'une chaîne encyclopédique, le fondement d'une reconstitution épique ou le prétexte d'une entreprise de moralisation. Mais aussi limité et partial soit-il, le recours à la compilation montre qu'elle a acquis un poids d'autorité littéraire et historique suffisant pour marquer, durant trois siècles au moins, le paysage intellectuel des cours de France et d'Italie.

Catherine Croizy-Naquet

Paris X-Nanterre

200, avenue de la République

92001 Nanterre cedex

e_naquet@club-internet.fr 